\title{
Antiangiogenic effects of pazopanib in xenograft hepatocellular carcinoma models: evaluation by quantitative contrast-enhanced ultrasonography
}

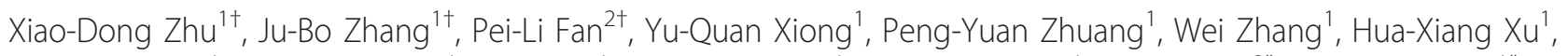
Dong-Mei Gao', Ling-Qun Kong ${ }^{1}$, Lu Wang ${ }^{1}$, Wei-Zhong Wu' ${ }^{1}$, Zhao-You Tang ${ }^{1}$, Hong Ding ${ }^{2^{*}}$, Hui-Chuan Sun ${ }^{{ }^{*}}$

\begin{abstract}
Background: Antiangiogenesis is a promising therapy for advanced hepatocellular carcinoma (HCC), but the effects are difficult to be evaluated. Pazopanib (GW786034B) is a pan-vascular endothelial growth factor receptor inhibitor, the antitumor effects or antiangiogenic effects haven't been investigated in HCC.

Methods: In vitro direct effects of pazopanib on human HCC cell lines and endothelial cells were evaluated. In vivo antitumor effects were evaluated in three xenograft nude mice models. In the subcutaneous HCCLM3 model, intratumoral blood perfusion was detected by contrast-enhanced ultrasonography (CEUS), and serial quantitative parameters were profiled from the time-intensity curves of ultrasonograms.

Results: In vitro proliferation of various HCC cell lines were not inhibited by pazopanib. Pazopanib inhibited migration and invasion and induced apoptosis significantly in two HCC cell lines, HCCLM3 and PLC/PRF/5. Proliferation, migration, and tubule formation of human umbilical vein endothelial cells were inhibited by pazopanib in a dose-dependent manner. In vivo tumor growth was significantly inhibited by pazopanib in HCCLM3, HepG2, and PLC/PRF/5 xenograft models. Various intratumoral perfusion parameters changed over time, and the signal intensity was significantly impaired in the treated tumors before the treatment efficacy on tumor size could be observed. Mean transit time of the contrast media in hotspot areas of the tumors was reversely correlated with intratumoral microvessel density.
\end{abstract}

Conclusions: Antitumor effects of pazopanib in HCC xenografts may owe to its antiangiogenic effects, and the in vivo antiangiogenic effects could be evaluated by quantitative CEUS.

\section{Background}

Hepatocellular carcinoma (HCC), one of the most vascularized solid cancers [1], is the sixth most common cancer and the third most common cause of death from cancer worldwide [2]. This disease is usually diagnosed at an advanced stage, and therefore it presents a dismal prognosis, owing to the lack of effective systemic treatment options and the underlying liver

\footnotetext{
* Correspondence: ding.hong@zs-hospital.sh.cn; sun.huichuan@zs-hospital.sh. $\mathrm{cn}$

+ Contributed equally

'Liver Cancer Institute and Zhongshan Hospital, Fudan University, Shanghai 200032, People's Republic of China

${ }^{2}$ Department of Ultrasound, Zhongshan Hospital, Fudan University, Shanghai 200032, People's Republic of China

Full list of author information is available at the end of the article
}

disease [3]. Overall survival may be improved after the clinical application of sorafenib or other molecular targeting agents [4,5]. Tumor angiogenesis plays a critical role in the development and progression of $\mathrm{HCC}$, which suggests that antiangiogenic therapy is effective in inhibiting tumor progression. The role of vascular endothelial growth factor (VEGF) and VEGF receptors (VEGFR) in the angiogenesis and progression of HCC has been well documented [6-8], providing a rationale for investigating VEGF or VEGFR-targeted therapies. Several clinical studies support antiangiogenic therapies in HCC, including bevacizumab $[9,10]$, sorafenib [5], and others [11]. With the advent of these antiangiogenic therapies, there is increased interest in determining the clinical effects of these agents. Given the

\section{Ciomed Central}

(c) 2011 Zhu et al; licensee BioMed Central Ltd. This is an Open Access article distributed under the terms of the Creative Commons Attribution License (http://creativecommons.org/licenses/by/2.0), which permits unrestricted use, distribution, and reproduction in any medium, provided the original work is properly cited. 
novel mechanism of action of these targeted agents, the classical definition of tumor response, tumor size reduction, may need to be revised. Thus there is a need for better understanding of how imaging methods may be utilized to assess the physiologic status of the tumor neovasculature and the activity of these agents in clinical use.

The change of intratumoral microvessel density (MVD) has been widely used to detect the response of antiangiogenic therapy in animal models, but its predictive value of treatment response was questioned in clinical settings because of some limitations [12,13]. For example, repeated biopsy to facilitate histological examination is usually impossible in clinical settings, especially in patients with HCC, which is often associated with coagulation dysfunction or ascites. Therefore noninvasive or minimally invasive methods is needed to monitor the biological response. Tumor perfusion measured by imaging methods, such as dynamic contrast-enhanced computed tomography (DCE-CT) and dynamic contrast-enhanced magnetic resonance imaging (DCE-MRI), have been reported [14-17]. With the use of microbubble contrast agents, contrastenhanced ultrasonography (CEUS) could also provide a sensitive method to detect tumor perfusion [18-21]. With more convenient application, the diagnostic value of CEUS is similar to DCE-CT and DCE-MRI [22,23]. CEUS is nowadays widely used in diagnosis [24] or in predicting the treatment efficacy of local therapies [25-27] in HCC, but assessment of the efficacy of the targeted therapies was scarcely reported. CEUS, combined with a computerized imaging analysis system, can depict the perfusion with serial parameters to quantitative blood perfusion in multiple characteristics by analysing the time-intensity curve (TIC) obtained from regions of interest (ROI). In two previous studies assessing angiogenesis [20] or efficacy of antiangiogenic therapy [19] with CEUS, the correlation between MVD and parameters of CEUS was controversial $[19,20]$, and only a few parameters were quantified, while the dynamic changes of the parameters during antiangiogenic therapy were not well observed.

In this study, the antitumor effect of pazopanib (GW786034B; GlaxoSmithKline, Collegeville, PA), a small-molecule tyrosine kinase inhibitor of VEGFRs, platelet-derived growth factor receptors, and c-Kit tyrosine kinases [28], were evaluated in 3 human HCC xenograft models. By analysis of dynamic images of CEUS, intratumoral blood perfusion was profiled by serial quantitative parameters, and biologic implications of these parameters were evaluated. Some CEUS parameters may be used as surrogate markers of MVD or used to quantitatively measure the intratumoral perfusion changes in the context of antiangiogenic treatment.

\section{Methods}

\section{Cell lines and cell culture}

Human HCC cell lines with high metastatic capacity (HCCLM3, MHCC97-H, and MHCC97-L, which were established by our institute [29]) or low metastatic capacity (SMCC7721, HepG2, and PLC/PRF/5) from ATCC cell bank were grown as a monolayer culture in Dulbecco's modified Eagle's medium or RPMI-1640 supplemented with $10 \%$ fetal calf serum, $100 \mathrm{U} / \mathrm{mL}$ penicillin, and $50 \mu \mathrm{g} / \mathrm{mL}$ streptomycin. Human umbilical vein endothelial cells (HUVEC, ScienCell Research Laboratories, San Diego, CA) and tumor-derived endothelial cells (TEC), established as previously described [30], were maintained on an endothelial cell medium (ScienCell Research Laboratories), and cells at passage 3 or 4 were chosen for experiments. All these cells were cultured at $37^{\circ} \mathrm{C}$ in a $5 \% \mathrm{CO}_{2}, 95 \%$ air environment in humidified incubators. Pazopanib, provided by GlaxoSmithKline in a purified powder, were reconstituted in dimethyl sulphoxide (DMSO) at a concentration of 300 $\mu \mathrm{g} / \mathrm{mL}$ before use.

\section{Proliferation assay and Apoptosis assays}

CellTiter-Glo ${ }^{\circledR}$ luminescent cell viability assay (Promega, Madison, WI) was used to test the proliferation of cells in response to different concentrations of pazopanib, following the manufacturer's instructions. Briefly, $2 \times$ $10^{3}$ cells, HUVEC, TEC, SMCC7721, HCCLM3, MHCC97-H, MHCC97-L, HepG2, and PLC/PRF/5 were plated in the 96-well opaque-walled multiwell plates in culture medium. Control wells containing medium without cells were set to obtain a value for background luminescence. After adherent culture, pazopanib (final concentrations of $0,1,2,5,10$, and $20 \mu \mathrm{g} / \mathrm{mL}$ ) was added to each well and incubated for $72 \mathrm{~h}$, and $80 \mu \mathrm{L}$ CellTiter-Glo reagent was then added in each well. After being mixed and incubated at room temperature for $10 \mathrm{~min}$, the luminescence of each well was recorded. HCCLM3, HepG2, and PLC/PRF/5 cells which were cultured in serum-free DMEM with $25 \mu \mathrm{g} / \mathrm{mL}$ pazopanib or vehicle for $6 \mathrm{~h}$ were collected and analysed for the presence of apoptotic cells using Annexin V-FITC Apoptosis Detection Kit (BD Pharmingen, San Jose, CA) following the manufacturer's instruction. Flow cytometry analysis was performed using FACS Calibur cytometer (R\&D Systems, Minneapolis, MN). Replicate assays were done.

\section{Tumor cell migration and invasion assay}

Transwell chamber inserts (Corning Inc, Corning, NY) with filter membrane pore size of $8 \mu \mathrm{m}$ were coated with $80 \mu \mathrm{L}$ Matrigel $(0.8 \mathrm{mg} / \mathrm{mL}$, BD Bioscience, Mountain View, CA). Tumor cells, HCCLM3, HepG2, and PLC/PRF/5, at the concentration of $5 \times 10^{5} / \mathrm{mL}$ 
serum-free DMEM were incubated with $25 \mu \mathrm{g} / \mathrm{mL}$ pazopanib or vehicle at the upper chamber. DMEM containing $10 \%$ FBS was added to the lower compartment. Seventy-two hours later, cells that migrated through the permeable membrane were fixed in paraformaldehyde, stained with Giemsa, and counted under an inverted light microscope at $100 \times$ magnification. Each assay was done at least in triplicate. Migration assay were applied similarly without coating the upper chamber with Matrigel, and the cells migrated through the membrane were counted at $48 \mathrm{~h}$.

\section{Wound-healing assay}

HUVEC were seeded in 24-well plates and incubated for $12 \mathrm{~h}$; the monolayer-adherent HUVEC were established. A line was scratched by a sterilized pin to wipe off the adherent cells in this line to create a wound. After being washed with PBS, pazopanib was then added to the medium (final concentrations of $0,1,4,20$, and $100 \mu \mathrm{g} / \mathrm{mL}$ ) and a control was set up with an equal concentration of DMSO. Seven hours later, the migration of HUVEC was assessed using an inverted light microscope at 50× magnification; the distances HUVEC migrated in the $7 \mathrm{~h}$ were measured.

\section{In vitro angiogenesis assay (tubule formation assay)}

In vitro formation of capillary-like structures was studied on growth factor-reduced Matrigel (BD Biosciences) diluted $1: 1$ on ice with cold endothelial cell medium in a 96-well plate. $5 \times 10^{4}$ HUVEC were inoculated on the surface of the Matrigel, after the adherent culture under normoxic conditions was reached and pazopanib (final concentrations of $0,10,20$, and $40 \mu \mathrm{g} / \mathrm{mL}$ ) was added. Tubule formation of HUVEC was assessed 3 and $6 \mathrm{~h}$ later under an inverted light microscope at $50 \times$ magnification.

\section{Animals}

Male BALB/c nu/nu mice (4-6 wk old) of around $20 \mathrm{~g}$ (Shanghai Institute of Materia Medica, Chinese Academy of Sciences, Shanghai) were housed in laminar-flow cabinets under specific pathogen-free conditions. The mice were cared for and handled according to the U.S. Public Health Service Policy on Humane Care and Use of Laboratory Animals. The experimental protocol was approved by the Shanghai Medical Experimental Animal Care Committee. Mice were inoculated subcutaneously in the right dorsal part near the lower pole of right kidney with $5 \times 10^{6}$ HCCLM3, which could induced high incidence of lung metastasis when implanted either subcutaneously or orthotopically in nude mice [29], or PLC/PRF/5 cells with low metastatic capacity. Orthotopic HepG2 xenograft model was established by implantation of tumor blocks at the average volume of $1 \mathrm{~mm}^{3}$.
Two weeks later, the xenograft model was established which was confirmed by conventional ultrasound with a high-frequency transducer; mice were then randomized into a pazopanib-treated group and a control group in a size-matched manner. Pazopanib was suspended in 0.5\% hydroxypropylmethyl cellulose (Sigma-Aldrich, St. Louis, MO) and $0.1 \%$ Tween-80 (Sigma-Aldrich) in water as a vehicle ( $\mathrm{pH} \mathrm{1.3-1.5)} \mathrm{and} \mathrm{was} \mathrm{given} 40 \mathrm{mg} / \mathrm{kg}$ daily by oral gavage. The control group received the vehicle alone at the same schedule and route of administration. Tumor burden was measured every week by ultrasound when CEUS was applied for subcutaneous HCCLM3 tumor model. Animals were sacrificed to obtain the tumors for histological study at the indicated time points. To evaluate the therapeutic effects of pazopanib, a pilot study was performed at a higher dosage $(60 \mathrm{mg} /$ $\mathrm{kg} / \mathrm{d}$ ) in HCCLM3 xenograft model in advance (Additional file 1, Figure S1).

\section{CEUS protocols}

The sonography investigators were blinded in regard to the groups. Animals of the subcutaneous HCCLM3 model were imaged on days $0,7,14$, and 21 after treatment. Conventional ultrasound and harmonic CEUS were performed using a commercially available ultrasound system iU22 (Philips, Bothell, WA). A L17-9 linear array transducer was used for the confirmation of planted tumor and the measurement of tumor volume, and a L9-3 linear array wideband transducer combined with Pulse Inversion Harmonic technique was used for CEUS study. Imaging settings, including mechanical index (0.1), frame rate $(18 \mathrm{~Hz})$, compress (36 dB), gain, focus, depth, and depth gain compensation line, were established after optimization at the beginning and were fixed in this study. Mice were anesthetized with sodium pentobarbital (50 mg/kg intraperitoneally) and placed in the prone position on a polystyrene plastic pad. The centrifuged ultrasound coupling gel was filled to the thickness of $5 \mathrm{~mm}$ for maintenance between the surface of the transducer and the skin of the tumor. To avoid the potential functional effects on the endothelial cells of the antiangiogenic agents [12], CEUS was performed at least $8 \mathrm{~h}$ (range, 8 - 12) after the daily administration of pazopanib. At each imaging session, tumor volumes were assessed by conventional B-mode imaging and calculated as $0.52 \times$ length $\times$ width $\times$ depth. During the contrast session, the longitudinal scan was chosen to display the largest dimension of the tumor and the right kidney. The transducer was then held in this position through the examination. Animals received a bolus injection of $0.02 \mathrm{~mL}(45 \mu \mathrm{g} / \mathrm{mL})$ microbubble contrast agent (SonoVue, Bracco SpA, Milan, Italy) within 1-2 s. The contrast medium consists of a suspension of sulphurhexafluoride microbubbles surrounded by a thin 
layer of phospholipid and palmitic acid that allows the microbubbles to withstand several passes through the pulmonary capillaries. Each bolus injection of SonoVue was through a 27 -gauge needle mounted to the tail vein, followed by a $0.2 \mathrm{~mL}$ saline flush. To control the quality of imaging, the enhancement in the imaging plane of the cortex of the ipsilateral kidney was also observed simultaneously. All the images were recorded in digital cine clips from the beginning of injection; recordings lasted for at least $2 \mathrm{~min}$ and were transferred in Digital Imaging and Communications in Medicine format to a PC for further analysis.

\section{Imaging data analysis}

Dynamic digital data sequences of CEUS were analysed off-line with the software SW-UCS (Shenwei Imaging Technology Company, Shanghai, China) by the sonography investigators. After browsing the dynamic perfusion of the whole tumor frame by frame, two ROIs were placed to generate two initial time-intensity curves (TICs) presenting dynamic changes of signal intensity in the selected region. One ROI was placed to cover the tumor area as a whole, and the other one was located in the most enhanced area in the tumor on CEUS, which was referred as a "hotspot" area. The shape of ROI was a circle drawn manually and the area of ROI approximately ranged from $5 \mathrm{~mm}^{2}$ to $800 \mathrm{~mm}^{2}$. By tracking every frame of contrast-enhanced images, the average intensity and initial TIC of each ROI were obtained. The initial TIC of each ROI was analysed and fitted with a least mean square method adaptable to bolus injection [31,32] and a series of quantitative parameters were calculated automatically with a mathematical func-

tion $I(t)=a_{0}+a_{1} \frac{e^{-a_{2} t}}{1+e^{-a_{3}\left(t-t_{0}\right)}}$, which was adaptable to the kinetics of bolus injection as reported by Postert, et al. [32] Serial quantitative parameters of each ROI achieved from fitted TIC solved at every pixel in each ROI were calculated automatically (Additional file 1, Figure S2). The obtained TIC typically presented a baseline phase prior to the contrast and then a rapid increase in the signal intensity to a peak level followed by a slow washout phase. Quantitative parameters derived from the fitted curve (Table 1), including increased signal intensity from baseline (ISI), and rate of signal increase (RSI) in the rising period, rate of washout of $50 \%$ contrast material (RWO), mean transit time (MTT) of the contrast material, area under curve $(\mathrm{AUC})$, and blood flow coefficient (BF; AUC/MTT $\times 2$ ). These parameters could be classified into two typesvolume related parameters, including AUC, ISI, and BF, and velocity related parameters, including RSI, RWO, and MTT. The volume related parameters were used to define the volume of blood perfusion in the ROI, while the velocity related parameters were used to define change of blood flow within a unit time in the area of interest. To reduce the variability among different subjects, which was probably due to the potential variation in the amounts of contrast entering the circulation, the volume-related parameters were standardized by dividing the corresponding parameters detected in the ipsilateral kidney and were presented as ratios.

\section{Histological study (necrosis, proliferation, hypoxia, and microvessel and pericyte density) in HCCLM3 models}

Thirty minutes after intraperitoneal injection with 60 $\mathrm{mg} / \mathrm{kg}$ pimonidazole hydrochloride (Chemicon International, Temecula, CA) [33,34], animals were anesthetized and infused via cardiac puncture with warm PBS followed by $4 \%$ paraformaldehyde/PBS. Tumors were dissected with the mark of scanning slice and direction on CEUS and fixed with $4 \%$ formaldehyde. The specimens were then dehydrated, embedded in paraffin, and sectioned at $6 \mu \mathrm{m}$. Sections were stained with $H \& E$ or were subjected to immunohistochemistry study, with primary antibodies against pimonidazole hydrochloride for hypoxia, CD31 (Abcam, Cambridge, MA) for tumor microvessels, and Ki-67 (Dako, Carpinteria, CA) for cell proliferation. Negative controls were treated identically but with the primary antibodies omitted. The components of the Envision-Plus detection system (EnVision/ HRP/Mo, Dako) were then applied. Reaction products were visualized by incubation with 3,3-diaminobenzidine. The necrosis index or hypoxia index was calculated as the ratio of necrotic area or pimonidazole stained area to the total area in the whole transverse section. MVD was determined by scanning stained tumor sections at low power, and areas of greatest CD31 positive density were chosen for analysis. The ratio of the positively stained cells to the total area of the field at the magnification of $200 \times$ was counted as MVD using Leica QWin Plus v3 software (Leica Microsystems Imaging Solutions, Cambridge, UK), as described previously [10]. The proliferation rate was counted as the proportion of the Ki-67-stained nuclei to the number of total nuclei in the field. MVD and proliferation rate were evaluated in at least 5 randomized chosen fields without necrosis within each tumor. Intratumoral pericyte density was evaluated in frozen sections by immunofluorescence staining with primary antibody against mouse $\alpha$-smooth muscle actin $(\alpha-$ SMA; Abcam) and Cy5-conjugated anti-rabbit IgG (Jackson ImmunoResearch, West Grove, PA) was used as the second antibody. $\alpha$-SMA-positive pericytes were counted in the graphics acquired at the magnification of $100 \times$ using an inverted fluorescence microscope. $\alpha$ SMA-positive tumor encapsulation was used as a 
Table 1 Terms used to portray the perfusion by contrast-enhanced ultrasonography

\begin{tabular}{|c|c|c|}
\hline Acronym & Parameter & Notes \\
\hline $\mathrm{TIC}$ & time-intensity curve & $\begin{array}{l}\text { A curve to describe the signal intensity of contrast media in a region of interest changes over time } \\
\text { when a series of ultrasonograms are analysed by specific software offline }\end{array}$ \\
\hline ROI & region of interest & $\begin{array}{l}\text { A circle drawn in the interested region for generating the time-intensity curve. In this study, one ROI } \\
\text { took the tumor region as a whole, the other one, "hotspot", was located in the highest enhanced area } \\
\text { on contrast-enhanced ultrasonography, which were determined by the two sonography investigators }\end{array}$ \\
\hline$|S|$ & increased signal intensity & The difference between baseline to the maximum increase in signal intensity in a region of interest \\
\hline RSI & rate of signal increase & The rate of signal enhancement from baseline to initial peak in a region of interest \\
\hline RWO & $\begin{array}{l}\text { rate of washout of } 50 \% \text { of } \\
\text { contrast material }\end{array}$ & The rate of signal intensity reduced to $50 \%$ of initial peak of contrast material in a region of interest \\
\hline MTT & mean transit time & Average persistence of a contrast material in a region of interest \\
\hline AUC & area under the curve & $\begin{array}{l}\text { Integral calculus of the area under time-intensity curve, which reflects the total perfusion of a region of } \\
\text { interest }\end{array}$ \\
\hline BF & blood flow coefficient & $\mathrm{BF}=\mathrm{AUC} / \mathrm{MTT} / 2$ \\
\hline
\end{tabular}

positive control, but was not calculated as pericytes when processing with Leica QWin Plus v3 software.

\section{Statistical Analysis}

Statistical comparisons were performed using the Student's $t$ test when data were normally distributed or using the nonparametric analyses of Spearman and Mann-Whitney when data were not normally distributed. One-way analysis of variance (ANOVA) was used to study the temporal variations of the CEUS parameters during tumor progression or during pazopanib treatment, and the Least Significant Difference test was used to compare between two time points, when necessary. Spearman's rank order correlation was used to investigate the relationships between tumor perfusion and histological features. Two-sided $P$ values less than 0.05 were considered statistically significant.

\section{Results}

\section{In vitro Studies}

The proliferation and survival of the tested human hepatoma cell lines were slightly affected by pazopanib at concentrations between 1 and $20 \mu \mathrm{g} / \mathrm{mL}$, whereas the proliferation of HUVEC and TEC was inhibited in a dose-dependent manner (Figure $1 \mathrm{~A}$ ), with an $\mathrm{IC}_{50}$ ranging between 5 and $15 \mu \mathrm{g} / \mathrm{mL}$ in $72 \mathrm{~h}$. Pazopanib could induce moderate apoptosis of HCCLM3 and $\mathrm{PLC} / \mathrm{PRF} / 5$ at the concentration of $25 \mu \mathrm{g} / \mathrm{mL}$, whereas the apoptosis of HepG2 cells was also not significantly enhanced by pazopanib (Figure 1B). Migration through the filter membrane of the Transwell chamber was significantly inhibited by pazopanib at the concentration of $25 \mu \mathrm{g} / \mathrm{mL}$ in HCCLM3, PLC/PRF/5, and HepG2 cells. The invasion through Matrigel could be also inhibited by pazopanib in these hepatoma cells except in HepG2 cells, which could not invade through the Matrigel even when treated with the vehicle only (Figure 1C). The migration of HUVEC was inhibited by pazopanib in a dose-dependent manner as detected by the wound-healing assay (Figure 1D). When the concentration reached $20 \mu \mathrm{g} / \mathrm{mL}$, the migration of the HUVEC was reduced by $30 \%$ compared with the vehicle-treated cells. Overwhelming cell death could be observed at the concentration of $100 \mu \mathrm{g} / \mathrm{mL}$, and the migration was difficult to be evaluated. Tubule formation of HUVEC was also inhibited in a dose-dependent manner by incubation with pazopanib for 3 or $6 \mathrm{~h}$. No tube was formed when the concentration reached $20 \mu \mathrm{g} / \mathrm{mL}$ or higher (Figure $1 \mathrm{E}$ ).

\section{Pazopanib inhibits xenograft tumor growth}

A pilot study showed that pazopanib (at a dose of 60 $\mathrm{mg} / \mathrm{kg}$ daily) significantly inhibited HCCLM3 growth and prolonged the survival of tumor-bearing mice (Additional file 1, Figure S1). To catch the perfusion changes in the course of treatment by weekly applied CEUS, instead of the changes merely at the end of treatment, a lower dosage of $40 \mathrm{mg} / \mathrm{kg} / \mathrm{d}$ was used in this study. After treated with pazopanib for $3 \mathrm{wk}$, the tumor growth of HCCLM3 was significantly delayed as compared with the control $\left(0.43 \pm 0.17 \mathrm{~cm}^{3}\right.$ versus $0.93 \pm$ $0.44 \mathrm{~cm}^{3}, \mathrm{n}=7$ and 5 for each group, $P=0.037$; Figure $2 \mathrm{~A})$. It is also noticed that the difference in tumor size (based on the 3 dimensions detected by ultrasonography in live animals) between the two groups was not statistically significant at the end of 1 wk or 2 wk after the initiation of the treatment $(0.16 \pm 0.08$ versus $0.20 \pm$ $0.13 \mathrm{~cm}^{3}, P=0.364 ; 0.35 \pm 0.21$ versus $0.45 \pm 0.23 \mathrm{~cm}^{3}$, $P=0.673$; respectively). Tumor growth were also significantly inhibited by 4-week pazopanib treatment in two other xenograft hepatoma models, including orthotopic HepG2 model $\left(0.64 \pm 0.16 \mathrm{~cm}^{3}\right.$ in control $[\mathrm{n}=6]$ versus $0.16 \pm 0.05 \mathrm{~cm}^{3}$ in pazopanib group [n $\left.=5\right], P=0.031$ ) and subcutaneous implanted PLC/PRF/5 model (0.86 \pm $0.32 \mathrm{~cm}^{3}$ in control versus $0.48 \pm 0.11 \mathrm{~cm}^{3}$ in pazopanib group, $\mathrm{n}=7$ for each group, $P=0.012$ ). 


\section{A}
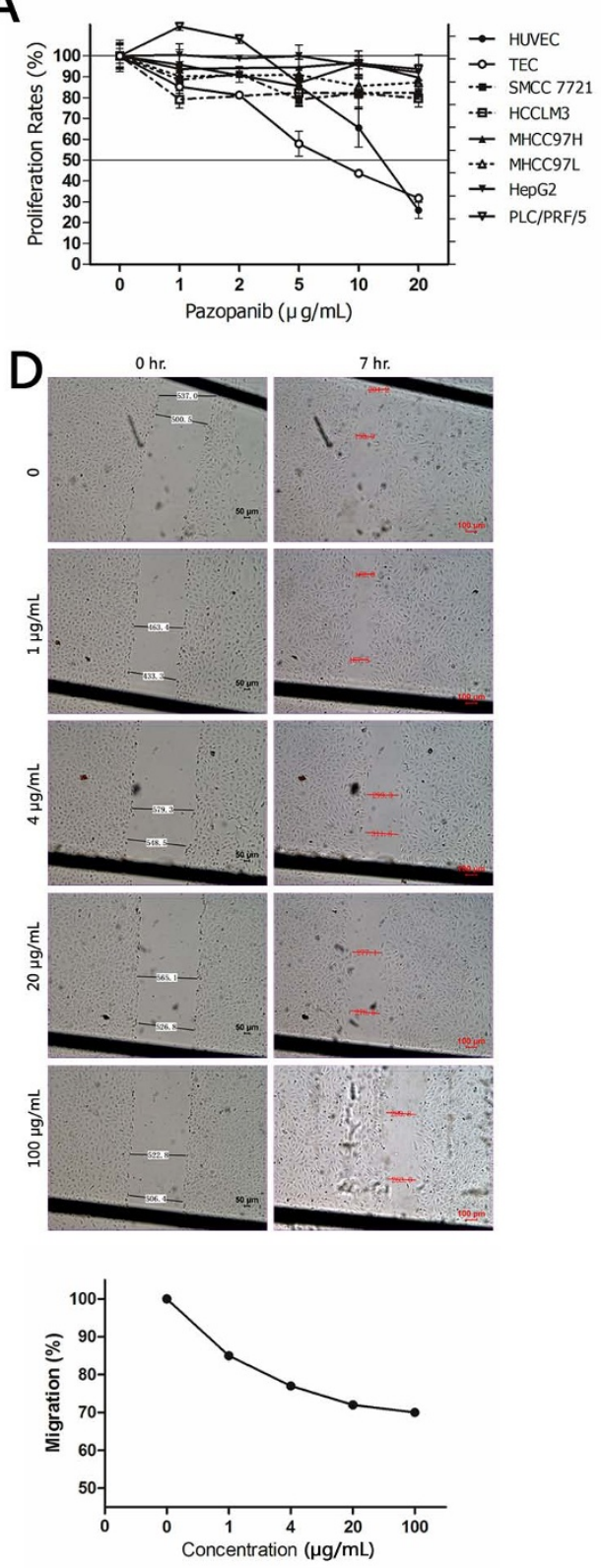

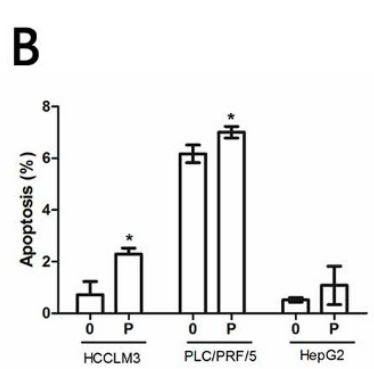

C

$\mathrm{E}$
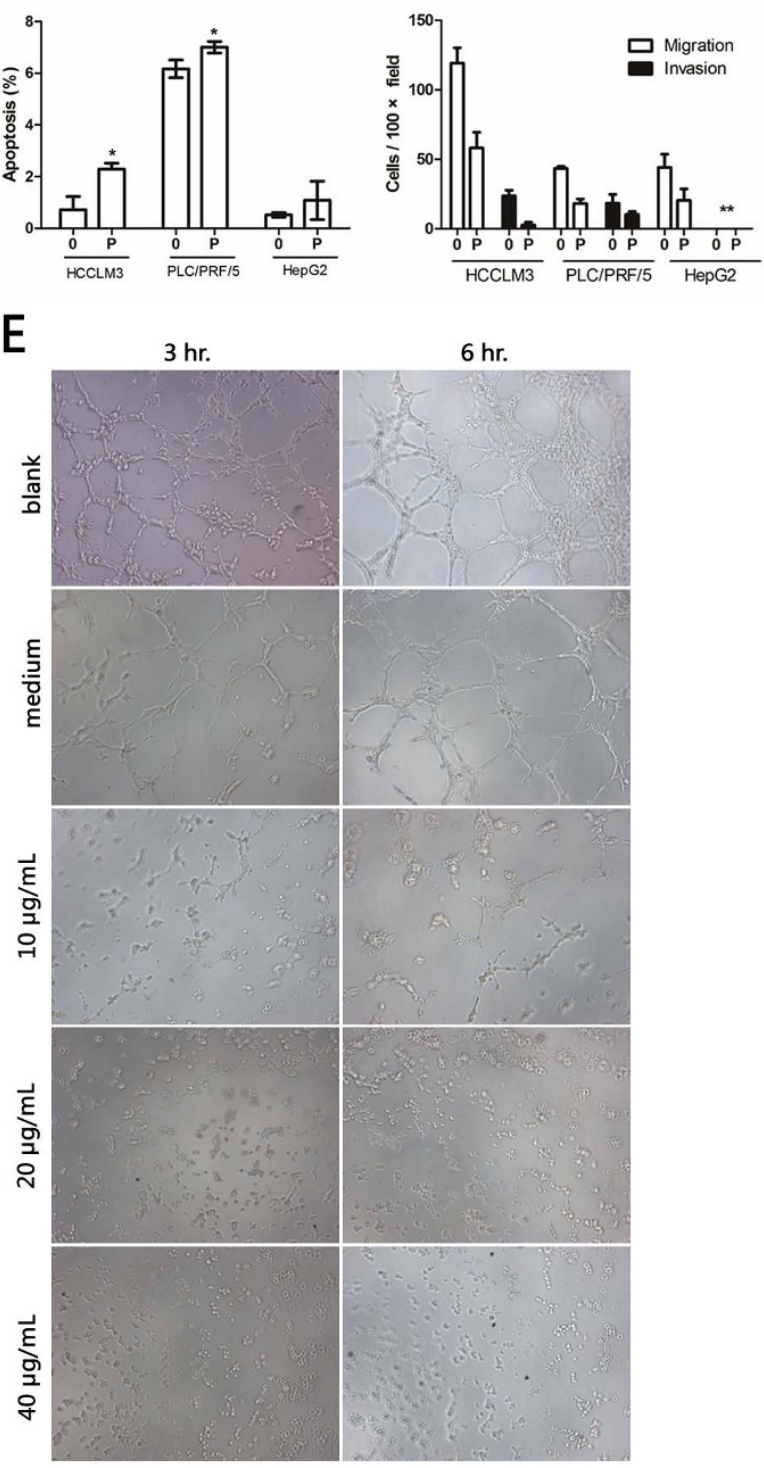

Figure 1 In vitro effects of pazopanib on hepatoma cells and endothelial cells. The effects of pazopanib on the in vitro proliferation of various human liver cancer cells, human umbilical vein endothelial cells (HUVEC) and tumor-derived endothelial cells (TEC) at various concentrations (A), and on the apoptosis (B) and migration and invasion of 3 liver cell lines (C) at the concentration of $25 \mu \mathrm{g} / \mathrm{mL}$. Tubule formation (D) and migration of HUVEC (E) were inhibited by pazopanib in a dose-dependent manner. Each experiment was repeated at least three times. Results of a representative study. Bars, SD; asterisks, $P<0.05$ as compared with control.

\section{Histological study in HCCLM3 models}

At the end of the third week, the mean MVD in the treated tumors was significantly lower than that in the control (3.1\% versus 5.8\%, $P=0.008$; Figure $2 \mathrm{~B}$ ). Endothelial cells formed dilated, disorganized, and tortuous networks in the control, while looking straight and regular in pattern in the pazopanib group (Figure 2B). Whereas the intratumoral $\alpha$-SMA-positive pericytes density in the pazopanib treated tumors was significantly higher than those in control $(3.5 \% \pm 0.8 \%$ versus $1.4 \% \pm 0.6 \%, P=0.001$; Figure $2 \mathrm{C}$ ). The necrosis and hypoxia indexes, evidenced by H\&E staining and pimonidazole immunostaining, were not significantly changed (64\% versus $67 \%, P=0.729$, and $34 \%$ versus $35 \%, P=$ 0.857 , respectively). The difference between the proliferation rates of the tumor cells in the pazopanib group 
A
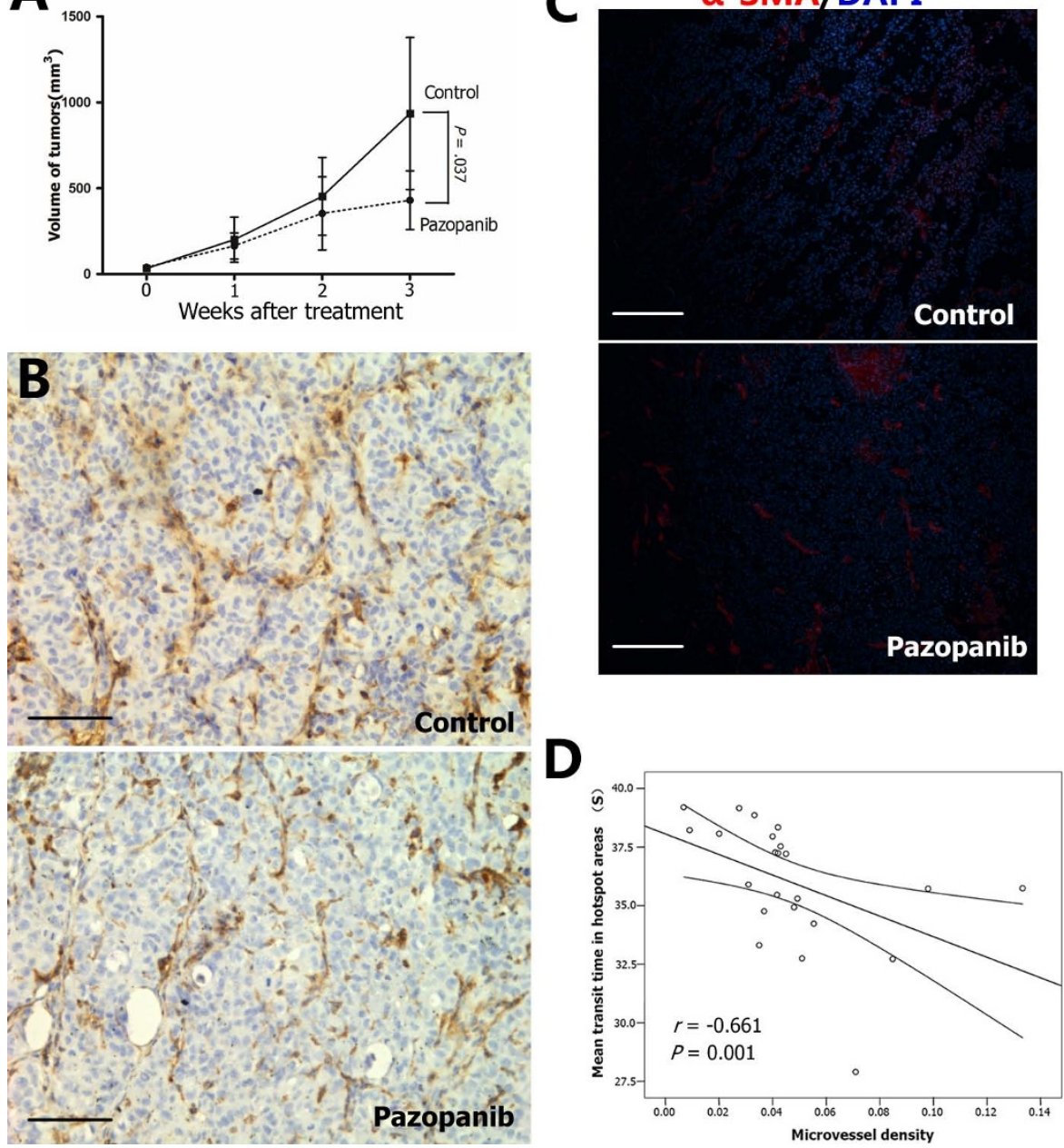

Figure 2 In vivo effects of pazopanib on the HCCLM3 xenograft model. Pazopanib inhibited in vivo tumor growth and intratumoral angiogenesis in a subcutaneously implanted HCCLM3 model. Three weeks after the treatment, tumor growth was significantly delayed as compared with the control (A). Microvessels evidenced by anti-CD31 immunostaining were lower in the pazopanib-treated tumors than the tumors treated with vehicle only (B). Endothelial cells formed dilated, disorganized, and tortuous networks in the control, while looking straight and regular in pattern in the pazopanib-treated tumors (B). The density of alpha-smooth muscular actin ( $\alpha$-SMA)-positive pericytes were increased by pazopanib treatment (C). The intratumoral microvessel density reversely correlated with the mean transit time of the contrast flowing through the xenograft tumors detected in the hotspot areas by contrast-enhanced ultrasonography (D). Bars, SD.

and the control was also not significant $(33.9 \%$ versus $28.1 \%, P=0.188)$. It should be noted that the tumor necrosis index correlated well with the tumor size $(r=$ $0.670, P<0.001)$. The tumor in the pazopanib-group was smaller in size but have similar necrosis index in compare with the control group, this may be resulted in the potential pro-necrotic effects of pazopanib. Although a significant correlation was also found between the hypoxia index and tumor size $(r=0.719, P<0.001)$, the standardized hypoxia index in the treated tumors was higher than in the untreated groups, but the difference was not significant $\left(0.8 \times 10^{-3} / \mathrm{mm}^{3}\right.$ versus $\left.0.4 \times 10^{-3} / \mathrm{mm}^{3}, P=0.092\right)$.

\section{Correlations between tumor perfusion and histological} features

Putting the two cohorts (the treated and untreated tumors) together, correlations between the histological features (e.g., necrosis and hypoxia index) obtained in the cross sections of the tumors and the parameters obtained by CEUS were evaluated. Two ROI were selected from each tumor, when the sonography was analysed. One ROI was placed to cover the entire tumor section as a whole, the other was located in the most enhanced area in CEUS, which was referred as a "hotspot" area. Terms used to portray the perfusion by 
Table 2 The correlations between the perfusion parameters measured by contrast-enhanced ultrasonography in the entire transverse tumor area and histological features $(n=12)$

\begin{tabular}{|c|c|c|c|c|c|c|c|}
\hline & & \multicolumn{3}{|c|}{$\begin{array}{l}\text { Velocity-related } \\
\text { parameters }\end{array}$} & \multicolumn{3}{|c|}{$\begin{array}{l}\text { Volume-related } \\
\text { parameters }\end{array}$} \\
\hline & & RWO & RSI & MTT & ISI & AUC & $\mathrm{BF}$ \\
\hline \multirow[t]{2}{*}{ Necrosis index } & $r$ & -0.423 & -0.656 & 0.139 & -0.482 & 0.156 & 0.096 \\
\hline & P & 0.028 & 0.000 & 0.491 & 0.011 & 0.436 & 0.634 \\
\hline \multirow[t]{2}{*}{ Hypoxia index } & $r$ & -0.470 & -0.656 & 0.08 & -0.332 & 0.269 & 0.212 \\
\hline & P & 0.024 & 0.001 & 0.72 & 0.122 & 0.215 & 0.330 \\
\hline \multirow[t]{2}{*}{ Microvessel density } & $r$ & -0.212 & 0.002 & -0.331 & 0.154 & 0.318 & 0.323 \\
\hline & $P$ & 0.287 & 0.990 & 0.092 & 0.444 & 0.106 & 0.100 \\
\hline
\end{tabular}

quantitative CEUS and their abbreviations and brief introductions were shown in Table 1.

As shown in Table 2, the necrosis and hypoxia indexes were correlated with some of the velocityrelated parameters obtained from the whole tumor (RSI and RWO) of entire tumor $(P<0.05)$. No significant correlation was found between volume-related parameters except ISI, obtained from the entire tumor area and the necrosis index. No significant correlation was found between MVD and all the parameters of CEUS in the entire tumor (Table 1). The correlations between the CEUS parameters from ROIs in the hotspot area and the histological features obtained from non-necrotic areas were evaluated as well (Additional file 1, Table S1). MVD was reversely correlated with MTT measured in the hotspot area $(r=-0.661, P=0.001$; Figure $2 D)$ in the two cohorts (including the treated and untreated tumors, $\mathrm{n}=12$ in total). And this correlation was true in the treated group $(r=-0.556, P=0.039, \mathrm{n}=7)$ but not in the control $(r=-0.367, P=0.332, \mathrm{n}=5)$. No statistically significant correlation was found between other histological features (necrosis and hypoxia indexes) or tumor size and all perfusion parameters detected in the hotspot area, except a borderline significant correlation between the necrosis index and BF detected in the hotspot area $(P=0.043$; Additional file 1 , Table S1).

Figure $3 \mathrm{~A}$ showed two typical sonograms and the TICs obtained from the kidney and entire tumor in the mice from the treated group and the control group at the end of the third week of the intervention, when intratumoral blood perfusion was depressed in the treated mice, whereas the blood perfusion of the ipsilateral kidney was slightly affected by pazopanib treatment (Additional file 1, Table S2). Figure 3B presented the perfusion changes of entire tumors in treated and control groups over time. Compared with the control, the volume-related parameters (ISI, AUC, and BF) of the entire tumor in treated tumors were significantly increased at the end of the first week. But at the end of the second week, when the tumor of the treated group was slightly smaller than that of the control (Figure 3B), all these parameters decreased, among which ISI was significantly lowered (Figure 3B). At the end of the third week, the tumor growth was significantly retarded in the treated group and all these parameters were significantly lowered in the pazopanib treated group (Figure 3B). On the other hand, the velocity-related parameters (RSI, RWO and MTT) in treated group changed gradually and were significant at the end of third week (Additional file 1, Table S3), especially the parameter of MTT, which was significantly prolonged at the end of the third week in the treated tumors (Figure 3B). These results indicated that 3-wk pazopanib treatment could lower the peak and the total perfusion of the tumor and could prolong MTT of the contrast material flowing through the tumor.

\section{Discussion}

In this study, we demonstrated the antiangiogenic effects of pazopanib in several human HCC xenograft models, which can be continuously and noninvasively assessed by quantitative CEUS. Oral administration of pazopanib at doses ranging from 10 to $100 \mathrm{mg} / \mathrm{kg}$ daily was previously shown to inhibit tumor growth in several human tumor xenograft models $[28,35]$. The effect of pazopanib on HCC, one of the most vascularized tumors, has not been reported before. Daily treatment with pazopanib at a dose of $40 \mathrm{mg} / \mathrm{kg}$ can effectively inhibit tumor growth of 3 different human HCC xenograft models and prolong the life span of tumor-bearing nude mice, which may attribute to the antiangiogenic effects of pazopanib. It remain controversy that tumor cells or endothelial cells, which cell type is main target of the tyrosine kinase inhibitors. In this study, direct anti-proliferation effect of pazopanib on hepatoma cells was not significant either in vitro or in vivo. Although in vitro pazopanib treatment could induce cell apoptosis and inhibit invasion and migration features in some tumor cells, the concentration of pazopanib is relatively high. To the contrary, we found pazopanib could inhibit the proliferation, migration, and tubule formation of HUVEC in vitro and could reduce the intratumoral MVD in vivo. So, the in vivo antitumor effects of pazopanib may be indirect and may attribute to its antiangiogenic effects, at least at the dosage of $40 \mathrm{mg} / \mathrm{kg} / \mathrm{d}$. Similar indirect antitumor effects were reported by Kumar et al. [28] in several other tumor models. Podar et al. [35] found that pazopanib can block multiple myeloma cell growth and survival in vitro. It may therefore be speculated that direct anti-proliferation effects of pazopanib on tumor cells may vary among cell lines.

Using a mathematic model to describe the TIC may reduce the subjectivity of ultrasonography examinations. 


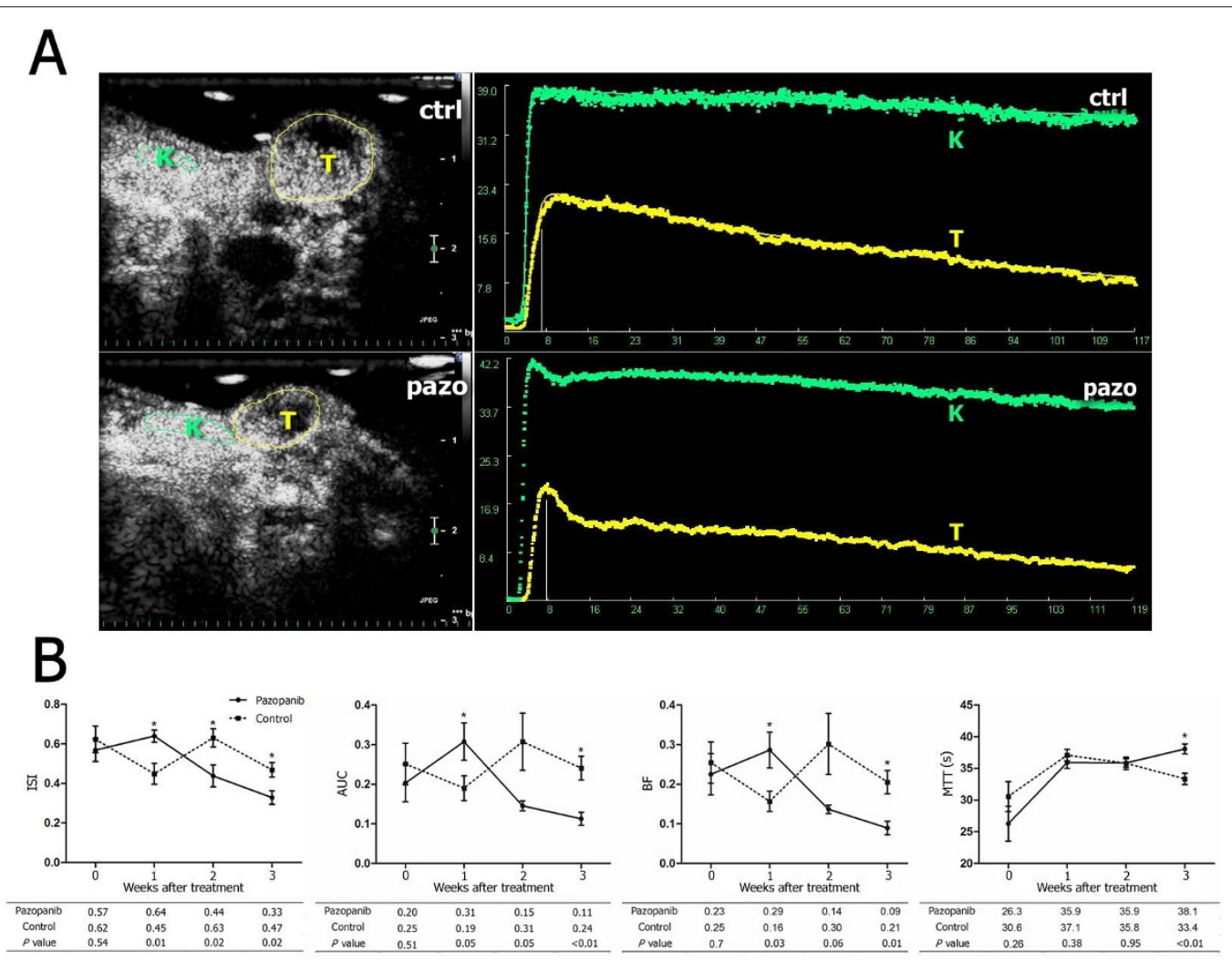

Figure 3 Intratumoral blood perfusion detected by quantitative contrast-enhanced ultrasonography in pazopanib-treated HCCLM3 xenograft model. Panel A shows typical images of the contrast-enhanced ultrasonography (CEUS) in subcutaneous tumor (T) and ipsilateral kidney (K) from the control (ctrl) and pazopanib-treated mice (pazo) 3 wk after the initiation of the treatment (left panels). ROls were selected in the tumor ("T," yellow lines) and ipsilateral kidney ("K," green lines), and the time-intensity curves (right panels) were then obtained in the two regions of interest for further analyses. Tumor perfusion changed in a biphasic manner (panel B). The standardized volume-related parameters detected by CEUS in the whole tumor changed over time. As compared to the control, an increase in signal intensity, the area under the curve, and the blood flow coefficient increased 1 wk after the pazopanib treatment, but decreased afterward. Mean transit time of the contrast in the tumor was also significantly prolonged at the end of the third week of the pazopanib treatment (A). Bars, SEM. Asterisks indicate $P<0.05$.

In the present study, serial parameters were obtained and could reflect the changes of tumor perfusion from different aspects (Table 1), from which ISI stood out. When the efficacy of pazopanib treatment was not proved by tumor size at the end of the second week (Figure 2A), the intratumoral peak perfusion (ISI) detected in the whole tumor started to drop and remained lower afterward, as compared with the control (Figure 3B). Meanwhile, the present study also showed ISI reversely correlated with tumor necrotic area, which was also increased in the treated tumor compared with the untreated tumor. This indicates that the therapeutic response of pazopanib could be sensitively detected by CEUS before a significant change in tumor size, and thus CEUS may possibly meet the demand for a novel method to define the biological response to targeted therapy, which usually did not reduce tumor size and therefore was not appropriate to be judged in traditional ways. The Choi criteria, which evaluated intratumoral necrosis by $\mathrm{CT}$, has been proposed recently [36]. In the present study, several parameters, including ISI obtained from the entire tumor, were found to be significantly correlated with the necrosis index. The necrotic tissue without perfusion reduced the average level of perfusion detected in the entire tumor, and therefore ISI obtained from the whole tumor reflects the mean level of the intratumoral peak perfusion and is affected by the necrosis index. ISI may be used as a comparable parameter in the Choi criteria in CEUS settings. However, ISI also reflects tumor perfusion in the nonnecrotic area, and seems better to compare with the evaluation of the necrosis area alone. The hypothesis of transient "normalizing tumor vasculature" following antiangiogenic therapy [37], whereby blood perfusion within an individual vessel, demonstrated as ISI, AUC, and BF in CEUS, may increase at the end of the first week (Figure 3B).

Another parameter, MTT, obtained from the ROI within the hotspot areas, was also highlighted. 
MTT obtained from these specific areas was the only parameter found to be associated with the MVD measured in the viable area (Figure 2D). MTT acquired from the entire tumor was also significantly prolonged at the third week in this study (Figure 3B). Although volume-related parameters (e.g., ISI) detected by CEUS were found to be associated with MVD by previous studies $[19,38,39]$, this correlation was not established in this study, probably due to the small sample size and different xenograft models. When microvessels in the viable areas lowered after antiangiogenic therapy, and then the flow-in and wash-out of the contrast was restricted, as a result, MTT, a measurement of the average persistence of a contrast material in the circulation [31], was prolonged. The biologic significance of MTT detected by CT perfusion scan was also revealed in a recently published study; its baseline and change following bevacizumab administration correlated with clinical outcome, whereas the volume parameters (e.g., blood flow and blood volume) did not [14]. When this correlation was further tested in subgroups, we found that the correlation was only established in the treated group. The immature and inefficient neovasculature in the tumor was more susceptible to the VEGF targeting therapy and could be pruned by eliminating excess endothelial cells, and the resulting vasculature is more functional [37]. In this study, the findings that endothelial cells looked straight and regular in pattern and that density of $\alpha$-SMA-positive pericytes was increased in the tumors from pazopanib-treated mice indicated that vessel normalization may be induced. And as an imaging technology capable of measuring the functional perfusion of contrast media, CEUS can only detect the functional vessels but not the new angiogenic sprouts which are always functionally invalid (not perfused by contrast media). And the new angiogenic sprouts were pruned by pazopanib and were dense in the control (Figure 2B). Further histological studies are needed to define the morphological and functional differences of the microvessels between the two groups and explain why the correlation between MTT and MVD could be found only in the treated group. More recently, Palmowski et al [40] evaluated large vessels and immature vessels with high-frequency Doppler ultrasound technique equipped by VisualSonics. Compared with the very high-frequency transducer which is only eligible for small animals, clinically available transducer used in the study had relatively lower resolution for tumor vessels. Although attempted, the CEUS system we used in this study could detect the blood perfusion of the subcutaneous tumors but not of the intrahepatic ones, therefore intratumoral perfusion of orthotopic HepG2 tumors were not detected in this study. However, the quantitative assessment of intratumoral perfusion is another method to detect the functional vessels in tumors treated with antiangiogenesis. The present study provides a rationale for testing the therapeutic effects of pazopanib or other targeted agents with antiangiogenic effects in patients with unresectable HCC.

This study has some limitations. First, although in vivo effects of pazopanib were evaluated in 3 human HCC nude mice models, other dosage and schedules of pazopanib or spontaneous model of liver cancer were not tested in this study. Second, fine changes of tumor perfusion may not be observed and only the subcutaneous tumor models could be detected by this equipment due to its relatively lower resolution and small tumor size. In the clinical settings, the tumors are always of large size, especially in the HCC patients with unresectable tumors which are the indications of antiangiogenic therapy. CEUS examination should be practical in these patients. Third, the burst/replenishment kinetics which had some advantages in minimizing the influence of cardiac output [41] was not used in our study. We took the perfusion of contrast in the ipsilateral kidney as a control for instead, which may also reduce the influence of cardiac output.

\section{Conclusions}

In humans, pazopanib is currently being studied in more than 40 clinical trials in various cancers, including a phase I study to evaluate the safety, tolerability, and optimal dosage in HCC patients. The findings of our study provide a rationale for testing the therapeutic effects of pazopanib in patients with unresectable HCC. As CEUS has several advantages over DCE-MRI and DCE-CT [42], the application of quantitative CEUS, especially the two parameters ISI and MTT, deserves to be further evaluated in comparative clinical trials, especially in much larger unresectable tumors.

\section{Additional material}

Additional file 1: Additional results including 2 figures and 3 tables Two figures (Figure S1-S2) and 3 tables (Table S1-S3) were included in this file.

\section{List of abbreviations used}

HCC: hepatocellular carcinoma; CEUS: contrast-enhanced ultrasonography; VEGF: vascular endothelial growth factor; VEGFR: VEGF receptor; MVD: microvessel density; HUVEC: human umbilical vein endothelial cells; DCE-CT: dynamic contrast-enhanced computed tomography; DCE-MRI: dynamic contrast-enhanced magnetic resonance imaging; DMSO: dimethyl sulphoxide; ANOVA: analysis of variance; TIC: time-intensity curve; ROI: region of interest; ISI: increased signal intensity; RSI: rate of signal increase; RWO: rate of washout of $50 \%$ contrast material; MTT: mean transit time; AUC: area under the curve; BF: blood flow coefficient.

\section{Acknowledgements}

This work was jointly supported by National Natural Science Foundation of China and the Research Grants Council (No. 30872409, 30872504, and 
30872505), "211" Project for Higher Education (211xk24-1), and National key science \& technology specific project (2008ZX10002-019, 021).

\section{Author details}

${ }^{1}$ Liver Cancer Institute and Zhongshan Hospital, Fudan University, Shanghai 200032, People's Republic of China. 'Department of Ultrasound, Zhongshan Hospital, Fudan University, Shanghai 200032, People's Republic of China.

\section{Authors' contributions}

HD and HCS participated in the design of the experiments, data interpretation, and manuscript preparing. $\mathrm{XDZ}, \mathrm{JBZ}$, and PLF equally contributed to this work. They performed the experiments, including in vitro experiments and histological studies (XDZ and JBZ) and in vivo experiments $(P L F)$, analyzed the data, and participated in writing of the manuscript. Ultrasonography was carried out by PLF and HD. YQX, PYZ, WZ, HXX, DMG, and LQK participated in the in vivo studies and histological studies. LW, WZW, and ZYT participated in the design of the experiments. All authors read and approved the final manuscript.

\section{Competing interests}

The authors declare that they have no competing interests.

Received: 19 May 2010 Accepted: 20 January 2011

Published: 20 January 2011

\section{References}

1. Sun HC, Tang ZY: Angiogenesis in hepatocellular carcinoma: the retrospectives and perspectives. J Cancer Res Clin Oncol 2004, 130(6):307-319.

2. Parkin DM, Bray F, Ferlay J, Pisani P: Global cancer statistics, 2002. CA Cancer J Clin 2005, 55(2):74-108.

3. Bruix J, Sherman M: Management of hepatocellular carcinoma. Hepatology 2005, 42(5):1208-1236.

4. Lopez PM, Villanueva A, Llovet JM: Systematic review: evidence-based management of hepatocellular carcinoma-an updated analysis of randomized controlled trials. Aliment Pharmacol Ther 2006, 23(11):1535-1547.

5. Llovet JM, Ricci S, Mazzaferro V, Hilgard P, Gane E, Blanc JF, de Oliveira AC, Santoro A, Raoul JL, Forner A, Schwartz M, Porta C, Zeuzem S, Bolondi L, Greten TF, Galle PR, Seitz JF, Borbath I, Haussinger D, Giannaris T, Shan M, Moscovici M, Voliotis D, Bruix J: Sorafenib in advanced hepatocellular carcinoma. N Engl J Med 2008, 359(4):378-390.

6. Semela D, Dufour JF: Angiogenesis and hepatocellular carcinoma. Journal of Hepatology 2004, 41(5):864-880

7. Pang RW, Poon RT: From molecular biology to targeted therapies for hepatocellular carcinoma: the future is now. Oncology 2007, 72(Suppl 1):30-44.

8. Liu Y, Poon RT, Li Q, Kok TW, Lau C, Fan ST: Both antiangiogenesis- and angiogenesis-independent effects are responsible for hepatocellular carcinoma growth arrest by tyrosine kinase inhibitor PTK787/ZK222584. Cancer Res 2005, 65(9):3691-3699.

9. Siegel $A B$, Cohen El, Ocean A, Lehrer D, Goldenberg A, Knox JJ, Chen $H$, Clark-Garvey S, Weinberg A, Mandeli J, Christos P, Mazumdar M, Popa E, Brown RS Jr, Rafii S, Schwartz JD: Phase II trial evaluating the clinical and biologic effects of bevacizumab in unresectable hepatocellular carcinoma. J Clin Oncol 2008, 26(18):2992-2998.

10. Zhu XD, Zhang JB, Zhuang PY, Zhu HG, Zhang W, Xiong YQ, Wu WZ, Wang L, Tang ZY, Sun HC: High expression of macrophage colonystimulating factor in peritumoral liver tissue is associated with poor survival after curative resection of hepatocellular carcinoma. J Clin Oncol 2008, 26(16):2707-2716.

11. Zhu AX: Development of sorafenib and other molecularly targeted agents in hepatocellular carcinoma. Cancer 2008, 112(2):250-259.

12. Ellis LM, Hicklin DJ: VEGF-targeted therapy: mechanisms of anti-tumour activity. Nat Rev Cancer 2008, 8(8):579-591.

13. Kerbel R, Folkman J: Clinical translation of angiogenesis inhibitors. Nature Reviews Cancer 2002, 2(10):727-739.

14. Zhu AX, Holalkere NS, Muzikansky A, Horgan K, Sahani DV: Early antiangiogenic activity of bevacizumab evaluated by computed tomography perfusion scan in patients with advanced hepatocellular carcinoma. Oncologist 2008, 13(2):120-125.
15. Wang $J H$, Min PQ, Wang PJ, Cheng WX, Zhang XH, Wang Y, Zhao XH, Mao XQ: Dynamic CT Evaluation of Tumor Vascularity in Renal Cell Carcinoma. AJR Am J Roentgenol 2006, 186(5):1423-1430.

16. Schlemmer HP, Merkle J, Grobholz R, Jaeger T, Michel MS, Werner A, Rabe J, van Kaick G: Can pre-operative contrast-enhanced dynamic MR imaging for prostate cancer predict microvessel density in prostatectomy specimens? Eur Radiol 2004, 14(2):309-317.

17. Hylton N: Dynamic contrast-enhanced magnetic resonance imaging as an imaging biomarker. J Clin Oncol 2006, 24(20):3293-3298.

18. Bertolotto M, Pozzato G, Croce LS, Nascimben F, Gasparini C, Cova MA, Tiribelli C: Blood flow changes in hepatocellular carcinoma after the administration of thalidomide assessed by reperfusion kinetics during microbubble infusion: preliminary results. Invest Radiol 2006, 41(1):15-21.

19. McCarville MB, Streck CJ, Dickson PV, Li CS, Nathwani AC, Davidoff AM: Angiogenesis inhibitors in a murine neuroblastoma model: quantitative assessment of intratumoral blood flow with contrast-enhanced grayscale US. Radiology 2006, 240(1):73-81.

20. Lucidarme O, Kono Y, Corbeil J, Choi SH, Golmard JL, Varner J, Mattrey RF: Angiogenesis: noninvasive quantitative assessment with contrastenhanced functional US in murine model. Radiology 2006, 239(3):730-739.

21. Bolondi L, Correas JM, Lencioni R, Weskott HP, Piscaglia F: New perspectives for the use of contrast-enhanced liver ultrasound in clinical practice. Dig Liver Dis 2007, 39(2):187-195.

22. Forner A, Vilana R, Ayuso C, Bianchi L, Sole M, Ayuso JR, Boix L, Sala M, Varela M, Llovet JM, Bru C, Bruix J: Diagnosis of hepatic nodules $20 \mathrm{~mm}$ or smaller in cirrhosis: Prospective validation of the noninvasive diagnostic criteria for hepatocellular carcinoma. Hepatology 2008, 47(1):97-104

23. Dai Y, Chen MH, Fan ZH, Yan K, Yin SS, Zhang XP: Diagnosis of small hepatic nodules detected by surveillance ultrasound in patients with cirrhosis: Comparison between contrast-enhanced ultrasound and contrast-enhanced helical computed tomography. Hepatol Res 2008, 38(3):281-290.

24. Lencioni R, Piscaglia F, Bolondi L: Contrast-enhanced ultrasound in the diagnosis of hepatocellular carcinoma. J Hepatol 2008, 48(5):848-857.

25. Bartolozzi C, Lencioni R, Ricci P, Paolicchi A, Rossi P, Passariello R: Hepatocellular carcinoma treatment with percutaneous ethanol injection: evaluation with contrast-enhanced color Doppler US. Radiology 1998, 209(2):387-393.

26. Catalano O, Esposito M, Lobianco R, Cusati B, Altei F, Siani A: Hepatocellular carcinoma treated with chemoembolization: assessment with contrast-enhanced doppler ultrasonography. Cardiovasc Intervent Radiol 1999, 22(6):486-492.

27. Cioni D, Lencioni R, Rossi S, Garbagnati F, Donati F, Crocetti L, Bartolozzi C: Radiofrequency thermal ablation of hepatocellular carcinoma: using contrast-enhanced harmonic power doppler sonography to assess treatment outcome. AJR Am J Roentgenol 2001, 177(4):783-788.

28. Kumar R, Knick VB, Rudolph SK, Johnson JH, Crosby RM, Crouthamel MC, Hopper TM, Miller CG, Harrington LE, Onori JA, Mullin RJ, Gilmer TM, Truesdale AT, Epperly AH, Boloor A, Stafford JA, Luttrell DK, Cheung M: Pharmacokinetic-pharmacodynamic correlation from mouse to human with pazopanib, a multikinase angiogenesis inhibitor with potent antitumor and antiangiogenic activity. Mol Cancer Ther 2007, 6(7):2012-2021

29. Li Y, Tian B, Yang J, Zhao L, Wu X, Ye SL, Liu YK, Tang ZY: Stepwise metastatic human hepatocellular carcinoma cell model system with multiple metastatic potentials established through consecutive in vivo selection and studies on metastatic characteristics. J Cancer Res Clin Oncol 2004, 130(8):460-468

30. Xiong $Y Q$, Sun $H C$, Zhang W, Zhu XD, Zhuang PY, Zhang JB, Wang L, Wu WZ, Qin LX, Tang ZY: Human hepatocellular carcinoma tumor-derived endothelial cells manifest increased angiogenesis capability and drug resistance compared with normal endothelial cells. Clin Cancer Res 2009, 15(15):4838-4846.

31. Cosgrove D, Eckersley R, Blomley M, Harvey C: Quantification of blood flow. Eur Radiol 2001, 11(8):1338-1344.

32. Postert T, Hoppe P, Federlein J, Helbeck S, Ermert H, Przuntek H, Buttner $T$ Wilkening W: Contrast agent specific imaging modes for the ultrasonic assessment of parenchymal cerebral echo contrast enhancement. $J$ Cereb Blood Flow Metab 2000, 20(12):1709-1716. 
33. Varia MA, Calkins-Adams DP, Rinker LH, Kennedy AS, Novotny DB, Fowler WC Jr, Raleigh JA: Pimonidazole: a novel hypoxia marker for complementary study of tumor hypoxia and cell proliferation in cervical carcinoma. Gynecol Oncol 1998, 71(2):270-277.

34. Raleigh JA, Chou SC, Arteel GE, Horsman MR: Comparisons among pimonidazole binding, oxygen electrode measurements, and radiation response in C3H mouse tumors. Radiat Res 1999, 151(5):580-589.

35. Podar K, Tonon G, Sattler M, Tai YT, Legouill S, Yasui H, Ishitsuka K, Kumar S, Kumar R, Pandite LN, Hideshima T, Chauhan D, Anderson KC: The smallmolecule VEGF receptor inhibitor pazopanib (GW786034B) targets both tumor and endothelial cells in multiple myeloma. Proc Natl Acad Sci USA 2006, 103(51):19478-19483.

36. Choi H, Charnsangavej C, Faria SC, Macapinlac HA, Burgess MA, Patel SR, Chen LL, Podoloff DA, Benjamin RS: Correlation of computed tomography and positron emission tomography in patients with metastatic gastrointestinal stromal tumor treated at a single institution with imatinib mesylate: proposal of new computed tomography response criteria. J Clin Oncol 2007, 25(13):1753-1759.

37. Jain RK: Normalizing tumor vasculature with anti-angiogenic therapy: a new paradigm for combination therapy. Nat Med 2001, 7(9):987-989.

38. Krix M, Kiessling F, Vosseler S, Farhan N, Mueller MM, Bohlen P, Fusenig NE, Delorme S: Sensitive noninvasive monitoring of tumor perfusion during antiangiogenic therapy by intermittent bolus-contrast power Doppler sonography. Cancer Res 2003, 63(23):8264-8270.

39. Magnon C, Galaup A, Rouffiac V, Opolon P, Connault E, Rose M, Perricaudet M, Roche A, Germain S, Griscelli F, Lassau N: Dynamic assessment of antiangiogenic therapy by monitoring both tumoral vascularization and tissue degeneration. Gene Ther 2007, 14(2):108-117.

40. Palmowski M, Huppert J, Hauff $P$, Reinhardt M, Schreiner $K$, Socher MA, Hallscheidt P, Kauffmann GW, Semmler W, Kiessling F: Vessel fractions in tumor xenografts depicted by flow- or contrast-sensitive threedimensional high-frequency Doppler ultrasound respond differently to antiangiogenic treatment. Cancer Res 2008, 68(17):7042-7049.

41. Krix M, Kiessling F, Farhan N, Schmidt K, Hoffend J, Delorme S: A multivessel model describing replenishment kinetics of ultrasound contrast agent for quantification of tissue perfusion. Ultrasound Med Biol 2003, 29(10):1421-1430.

42. Sessa C, Guibal A, Del Conte G, Ruegg C: Biomarkers of angiogenesis for the development of antiangiogenic therapies in oncology: tools or decorations? Nat Clin Pract Oncol 2008, 5(7):378-391.

\section{Pre-publication history}

The pre-publication history for this paper can be accessed here: http://www.biomedcentral.com/1471-2407/11/28/prepub

\section{doi:10.1186/1471-2407-11-28}

Cite this article as: Zhu et al:: Antiangiogenic effects of pazopanib in xenograft hepatocellular carcinoma models: evaluation by quantitative contrast-enhanced ultrasonography. BMC Cancer 2011 11:28.

\section{Submit your next manuscript to BioMed Central and take full advantage of:}

- Convenient online submission

- Thorough peer review

- No space constraints or color figure charges

- Immediate publication on acceptance

- Inclusion in PubMed, CAS, Scopus and Google Scholar

- Research which is freely available for redistribution

Submit your manuscript at www.biomedcentral.com/submit
Biomed Central 